\title{
Spin Torque Oscillator Based BFSK Modulation
}

\author{
R. Ma*, A. Purbawati ${ }^{\dagger}$, M. Kreißig*, F. Protze*, A. Ruiz-Calaforra ${ }^{\dagger}$, J. Hem ${ }^{\dagger}$, U. Ebels ${ }^{\dagger}$, F. Ellinger* \\ *Chair for Circuit Design and Network Theory \\ Technische Universität Dresden, 01062 Dresden, Germany \\ †Univ. Grenoble Alpes, CEA, INAC-SPINTEC, GNRS \\ SPINTEC F-38000 Grenoble, France
}

\begin{abstract}
This work presents a spin torque nano-oscillator (STNO) based binary frequency shift keying (BFSK) modulation schema implemented on a printed circuit board (PCB). Maximal input data rate reaches $20 \mathrm{Mbit} / \mathrm{s}$. Depending on the STNO used, carrier frequency can range from 1 to $10 \mathrm{GHz}$. Both DC and AC currents flowing through the STNO can be tuned between 0 to $4 \mathrm{~mA}$. Using one magnetic tunnel junction (MTJ) STNO, a $380 \mathrm{MHz}$ frequency shift around the center frequency $9 \mathrm{GHz}$ was observed, when the modulated current was toggled between $1.06 \mathrm{~mA}$ and $1.2 \mathrm{~mA}$ at a rate of $20 \mathrm{Mbit} / \mathrm{s}$. The PCB with a size of $50 \times 55 \mathrm{~mm}$ consumes maximal $130 \mathrm{~mA}$ at a $5 \mathrm{~V}$ supply.

This is the first work demonstrating that the STNOs are applicable for BFSK modulation on the wireless application level.
\end{abstract}

Index Terms-Spin Torque Nano Oscillator, Binary Frequency Shift Keying, Wireless Communication

\section{INTRODUCTION}

The huge potential of wireless sensor networks (WSNs) to control, monitor and communicate information is driving research on wireless communication schemes that need to be compact, low cost and low power. Spintronic devices are nanoelectronic devices based on ferromagnetic material heterostructures that make use of electron spin to convey functionalities to nanolelectronics devices. For instance, they provide solutions for fast and non-volatile random access memories. Such spintronic devices are compatible with CMOS technologies and are therefore regarded as a key building block for Systems-on-Chip in the future [1].

Recent studies have shown that spintronic devices can also provide solutions for compact frequency tunable microwave signal sources. They have the capability to convert a DC current of a few $\mathrm{mA}$ into an RF output voltage signal at base frequencies that cover a large range of $0.2-20 \mathrm{GHz}$. Since the frequency can be tuned around the base frequency via the injected DC current, it is also possible to encode information via frequency shift keying (FSK) onto the carrier signal. The aim here is to demonstrate the possibility of using spintronics for FSK in wireless communications compatible with the needs of WSNs. The frequency range targeted is 5$10 \mathrm{GHz}$ with bit rates of 10-100 Mbit/s.

\section{A. Spin Torque Nano Oscillator}

The spintronic devices used here are magnetic tunnel junction nanopillars that consist of a polarizing magnetic layer which is the fixed layer, an $\mathrm{MgO}$ magnetic tunnel barrier and a second ferromagnetic layer which is the free layer. The

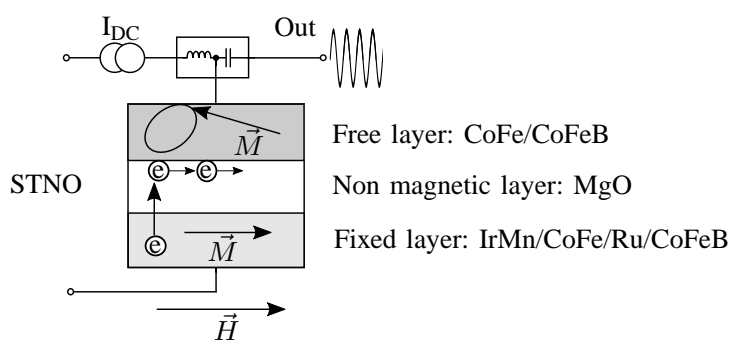

Fig. 1: STNO under test, its composition and working effect. Thickness of each layer in $\mathrm{nm}$ is as follows: $\mathrm{MgO}(1)$, $\mathrm{CoFe}(0.5) / \mathrm{CoFeB}(3.4), \operatorname{IrMn}(7) / \mathrm{CoFe}(1.8) / \mathrm{Ru}(0.4) / \mathrm{CoFeB}(2)$.

conversion of a DC current into an RF voltage signal makes use of two fundamental spintronic concepts, which are the spin momentum transfer and the tunneling magneto-resistance, shown in figure 1 . The current which is spin polarized by the fixed layer can transfer a spin angular momentum to the free layer. This results in a torque that compensates intrinsic damping and induces steady state oscillations of the magnetization, when the current is larger than a certain critical current value. The magneto-resistance effect describes the variation of the resistance when the angle between the free and fixed layer changes. The maximum value occurs between the parallel and antiparallel orientation. Due to the magneto-resistance, the magnetization oscillations are converted into voltage oscillations. As mentioned, the current value determines the oscillation frequency and permits besides the generation of an RF signal also its direct modulation, by modulating the current period. This effect will be used in this work.

In this work, the magnetic tunnel junction devices used are in-plane magnetized. This magnetization configuration leads to dynamic excitations at frequencies that lie in the range of 2-20 GHz, depending on the magnetic in-plane field. Our frequency target is $5-10 \mathrm{GHz}$. The typical device resistance is $200-500 \Omega$, the output power is around $-50 \mathrm{dBm}$ and the frequency tune-ability by current has a few hundred $\mathrm{MHz}$ per $\mathrm{mA}$. The design of the circuit has considered these values.

\section{B. State of the Art}

In the past years several studies have been performed on standalone devices to investigate the modulation properties of STNOs, using sinusoidal modulating currents by Quinsat et al. [2]. FSK has been first reported by Manfrini et al. [3] using vortex-based STNOs that emit in the 0.1-2 GHz range 

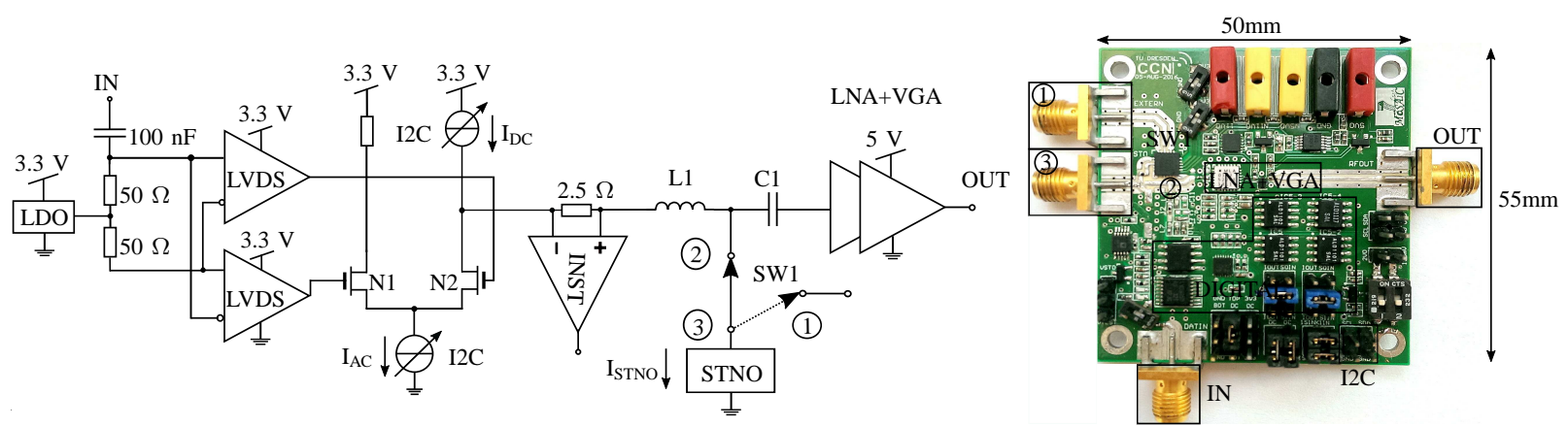

Fig. 2: (a) Simplified schematic of the STNO based BFSK modulation (b) Fabricated STNO based BFSK PCB.

by modulating the current between two values at data rates of $0-10 \mathrm{Mbit} / \mathrm{s}$. A first demonstration of OOK based wireless communications over 1 meter distance using STNOs has been done by Choi et al. [4]. Moreover, the MTJ STNO based ASK for a wireless communication system was studied by Sharma et al. [5]. These experiments were realized on standalone devices. The first demonstration of an STNO array based, OOK frequency modulation on a PCB level was achieved by Oh et al. [6].

The aim of this project is to demonstrate on the PCB level the BFSK wireless communication using spintronic materials for a transmitter and a novel concept for its receiver. Here the first steps undertaken will be presented for the realization of the transmitter to achieve the STNO based BFSK.

\section{Circuit Design}

Figure 2 shows the schematic of the STNO based BFSK modulation and its fabricated PCB respectively. As can be seen from the two figures, the design is composed mainly of two parts, a digital part which generates the two discrete current values, as well as an RF part which amplifies the AC signal coming out of the STNO. Between the digital and RF part there is a bias tee (composed by $\mathrm{L} 1=5 \mathrm{nH}$ and $\mathrm{C} 1=100 \mathrm{pF}$ ) blocking the $\mathrm{DC}$ and coupling the $\mathrm{AC}$ between the two parts. A micro-controller $(\mu \mathrm{C})$ board with an $\mathrm{I} 2 \mathrm{C}$ interface and a selfwritten Python program were used to enable a user-friendly control of the PCB.

The digital part consists of a differential CMOS transistor pair $\mathrm{N} 1$ and $\mathrm{N} 2$, and two current sources $\mathrm{I}_{\mathrm{AC}}$ and $\mathrm{I}_{\mathrm{DC}}$. Output currents of the two current sources are controllable by the $\mu \mathrm{C}$ board via its I2C interface. The differential pair is controlled by digital binary data coming into the SMA connector IN. Because the digital signal is usually a low voltage differential signal (LVDS), two LVDS-to-CMOS modules are used to convert the input LVDS into CMOS signals that control the CMOS differential pair. During working, only one of the differential pair is on, and two currents flowing through the STNO are obtained: (1) $\mathrm{I}_{\mathrm{STNO}}=\mathrm{I}_{\mathrm{DC}}$ when N1 is on and N2 off, (2) $I_{S T N O}=I_{D C}-I_{A C}$ when N1 is off and N2 on. Thus the current flowing through the STNO is shifted between two values $I_{D C}$ and $I_{D C}-I_{A C}$. Both $I_{D C}$ and $I_{A C}$ are tunable through the $\mu \mathrm{C}$ board in the range between 0 to $4 \mathrm{~mA}$ with a step of $0.033 \mathrm{~mA}$, according to the STNO characteristics (resistance, emitted frequency and etc.). To easily debug and verify $\mathrm{I}_{\mathrm{STNO}}$, an instrumentation amplifier (INST) with a fixed voltage gain of 4 is used. It is connected to a shunt resistor of $2.5 \Omega$ through which ISTNO flows.

The RF part consists of a DC-RF switch (SW1) with an insertion loss of $-1.3 \mathrm{~dB}$ over $0-8 \mathrm{GHz}$, a low noise amplifier (LNA) with a $-3 \mathrm{~dB}$ bandwidth of $10 \mathrm{GHz}$ and $15 \mathrm{~dB}$ gain as well as a variable gain amplifier (VGA) with a $-3 \mathrm{~dB}$ bandwidth of $20 \mathrm{GHz}$ and $10 \mathrm{~dB}$ gain.

The magnetic tunnel junction devices are nano-pillars with diameters in the range of $100 \mathrm{~nm}$. Due to process variation in nano-fabrication of the STNOs, individual devices can emit at different frequencies and have different qualities regarding tuning and frequency stability. In order to select appropriate devices from a chip, the switch SW1 with three ports (1), (2) and (3) is added to the board, so that the devices can be investigated first by lab equipment before they are connected to the digital and analog part of the board.

\section{EXPERIMENTAL RESULTS}

In the following, characterization methods of both the single PCB and the STNO devices are elaborated as well as the STNO based BFSK modulation study.

\section{A. Characterization of the $P C B$}

In order to define the maximum input data rate that the digital part of the PCB can handle, we replaced the STNO by a $270 \Omega$ resistor connecting ports (3) and (2) of SW1. The LNA and VGA were turned off. The modulation signal was a squared voltage pulse generated by a signal generator injected into the PCB's SMA connector IN. The currents were set to $\mathrm{I}_{\mathrm{DC}}=1.5 \mathrm{~mA}$ and $\mathrm{I}_{\mathrm{AC}}=1.5 \mathrm{~mA}$. The SMA connector (3) was connected to an oscilloscope and the eye diagram of the voltage over the resistor was monitored, as shown in figure 4. From this it is seen that the data rate can reach up to $20 \mathrm{Mbit} / \mathrm{s}$. The main factor that limits the data rate lies in an RC (dis)charging path composed by the STNO's resistance $\mathrm{R}_{\mathrm{STNO}}$ and the parasitic capacitance in the output capacitance of $\mathrm{I}_{\mathrm{DC}}$ and $\mathrm{N} 2$. It can be predicted, with the smaller $\mathrm{R}_{\mathrm{STNO}}$, the BFSK modulation rate can be $>20 \mathrm{Mbit} / \mathrm{s}$.

In order to test the RF part, we used a vector network analyzer Anritsu 37397D to register the S-parameters of the RF path between the SMA connector (3) and OUT in the 


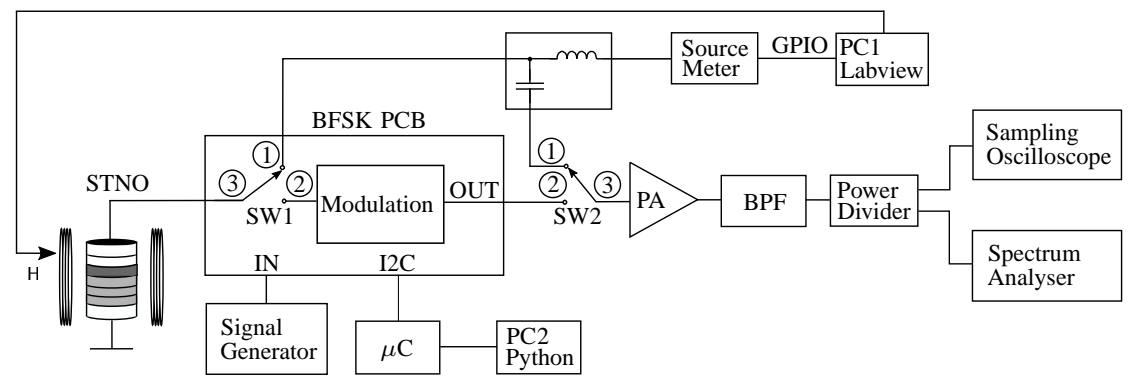

Fig. 3: Experimental setup for STNO based BFSK modulation. During STNO characterization, point (3) of switch SW1 and SW2 was connected to (1) of the corresponding switch. During BFSK modulation, (3) of SW1 and SW2 was connected to (2).

frequency range of $40 \mathrm{MHz}$ to $12 \mathrm{GHz}$, when both current sources were turned off. From the S21 we determined a $15 \mathrm{~dB}$ gain with a $-3 \mathrm{~dB}$ bandwidth of $4 \mathrm{GHz}$.

\section{B. Characterization of an STNO}

As already detailed in figure 1, the MTJ nanopillars have the following stack composition: freelayer/MgO/polarizer. The polarizer or the fixed layer is a synthetic anti-ferromagnet pinned by IrMn. Its structure is chosen, so that the $\mathrm{CoFeB}$ layer magnetization orientation remains fixed and stable. The nanopillar of elliptical shape $(65 \times 135 \mathrm{~nm})$ is embedded into coplanar waveguide electrodes that permit injection of a current perpendicular to the layers. Electrical contacts to the device are made using a ground-power-ground probe. The experimental set-up is shown in figure 3 .

In order to test the characteristics of the free running device, ports (1) and (3) of SW1 and SW2 were connected. In this way, a DC current was injected using a Keithley 2401 source meter, that also measured the DC voltage drop over the STNO from which the resistance was obtained. The DC current was injected into the STNO via the inductive part of an external bias tee, and the RF signal was extracted via the capacitive part. This RF signal was then amplified using an external power amplifier Miteq AMF-5D, connected to a 3-10 GHz bandpass filter. A power divider split the signal which was monitored by a sampling oscilloscope Textronix DPO72004 and a spectrum analyzer Agilent PNAE8363B. The device was placed into the gap of an electromagnet whose field can be swept between +/-1000 Oe. The DC current source and power supply of the electromagnet were computer controlled using a self-written code.

In a first step the magneto-resistance loop was obtained at a low current value of $0.05 \mathrm{~mA}$, which showed the switching of

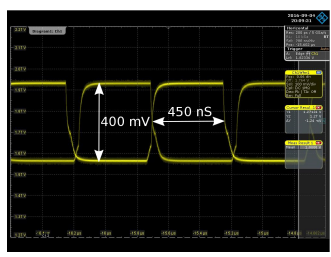

(a) Data rate $=2 \mathrm{Mbit} / \mathrm{s}$

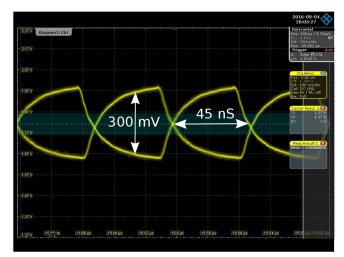

(b) Data rate $=20 \mathrm{Mbit} / \mathrm{s}$
Fig. 4: Voltage drop over the modeled STNO the magnetization of the free layer between the two resistance values of $151 \Omega$ (the parallel state) and $210 \Omega$ (the antiparallel state). The dynamic excitations occur for the antiparallel configuration. This sets the maximum current to be applied to $1.4 \mathrm{~mA}$, given a breakdown voltage of $300 \mathrm{mV}$ for these devices, as determined from other measurements. In order to find the operational conditions of current and field that yield steady state oscillations of the magnetization, the current and field were swept and the output signal from the spectrum analyzer was analyzed in view of its linewidth and power. Low linewidth $(<100-200 \mathrm{MHz})$, large output powers $(>10 \mathrm{nW})$ and a large $\mathrm{d} f / \mathrm{dI}$ are desired for a successful frequency modulation. For the given device, the best operation was obtained for a field value of $\mathrm{H}=780 \mathrm{Oe}$. The frequency, linewidth and power versus applied current $\mathrm{I}_{\mathrm{DC}}$ are given in figure 5. The data reveal that steady oscillations appear in the range of $0.7-1.2 \mathrm{~mA}$, where the frequency varies between 9 and $9.5 \mathrm{GHz}$, the linewidth is below $200 \mathrm{MHz}$, the power is $20-100 \mathrm{nW}$ and the slope $\mathrm{d} f / \mathrm{I}_{\mathrm{DC}}$ is $-530 \mathrm{MHz} / 0.5 \mathrm{~mA}$. As can be seen, the frequency decreases as a function of current, which is a specific signature of the in-plane magnetized STNO configuration. Furthermore, a higher current gives a higher output peak power, while the peak linewidth increases. This is undesired for modulation applications. However, it did not affect the demonstration of the BFSK modulation as will be shown in the next section.

\section{STNO Based BFSK Modulation}

In order to test the BFSK modulation, ports (3) and (2) were connected of both SW1 and SW2. Values of the DC current $\mathrm{I}_{\mathrm{DC}}$ and the $\mathrm{AC}$ current $\mathrm{I}_{\mathrm{AC}}$ were set via the $\mu \mathrm{C}$ board using a computer PC2 with the self-written Python program. The modulation data rate was varied between 1 to $20 \mathrm{Mbit} / \mathrm{s}$. For each value of modulation rate, the AC current was swept from 0 to $0.4 \mathrm{~mA}$ and the STNO output signal was registered on the spectrum analyzer and the oscilloscope.

Figure 6 shows the corresponding PSDs versus the AC current $\mathrm{I}_{\mathrm{AC}}$. A main frequency peak at around $9 \mathrm{GHz}$ is observed as expected for $\mathrm{I}_{\mathrm{DC}}=1.2 \mathrm{~mA}$ and $\mathrm{I}_{\mathrm{AC}}=0$. No second peak appears when $\mathrm{I}_{\mathrm{AC}}$ is smaller than $0.05 \mathrm{~mA}$, since the linewidth is broader than the induced frequency shift. With increasing $\mathrm{I}_{\mathrm{AC}}$, the second peak becomes visible at higher frequency, because $I_{S T N O}$ is shifted between $I_{D C}$ and $I_{D C}$ - 


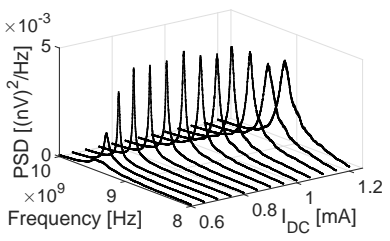

(a)

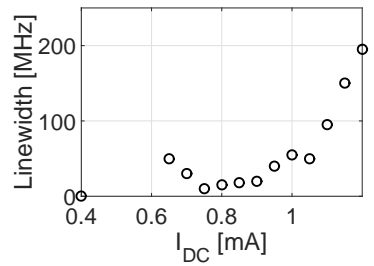

(c)

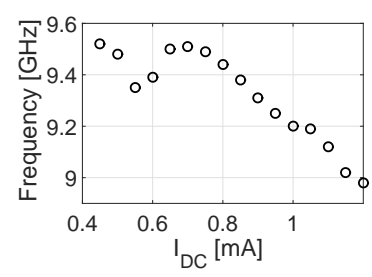

(b)

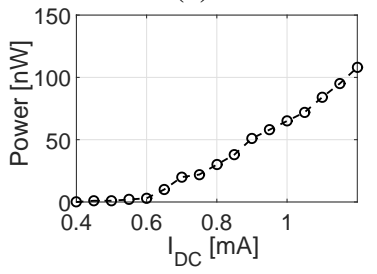

(d)
Fig. 5: (a) Oscillation frequency in dependence of $I_{D C}(b)$ Oscillation frequency versus $I_{D C}$ (c) Linewidth of frequency peak versus $I_{D C}(d)$ Power of frequency peak versus $I_{D C}$

$\mathrm{I}_{\mathrm{AC}}$ and the $\mathrm{d} f / \mathrm{dI}$ slope of these devices is negative, as is shown in figure 5 (b). The appearance of the second peak demonstrates that the STNO frequency shifts between two values, corresponding to the two current levels, which is in accordance with the $f$ versus I curve in figure 5 (a). This is a first proof for the BFSK modulation.

In a final step, we analyzed the time traces registered on the oscilloscope. The STNO output voltage is shown in figure 7 (a), after numerical filtering using a Matlab IIR filter with a $8-10 \mathrm{GHz}$ bandwidth. Here the current values were: $\mathrm{I}_{\mathrm{AC}}=0.14 \mathrm{~mA}$ and $\mathrm{I}_{\mathrm{DC}}=1.2 \mathrm{~mA}$ when data rate $=$ $20 \mathrm{Mbit} / \mathrm{s}$. From the voltage signal, the frequency shifts cannot be directly observed. However, a strong modulation of the amplitude modulation is clearly seen, which is in accordance with the dependence of the power versus current shown in figure 5 (d). In order to analyze the frequency shift, we used the Hilbert transformation to extract the amplitude and phase $\phi(\mathrm{t})$. The instantaneous frequency $f_{i}$ of the signal is then obtained from the derivative $f_{i}=\mathrm{d} \phi / \mathrm{dt}$. To reduce noise, we used the Savitzky-Golay frequency smoothing algorithm, with an average over 1000 points (corresponding to $10 \mathrm{~ns}$, less than the bit length $50 \mathrm{~ns}$ ). From figure 7 (b) it is seen

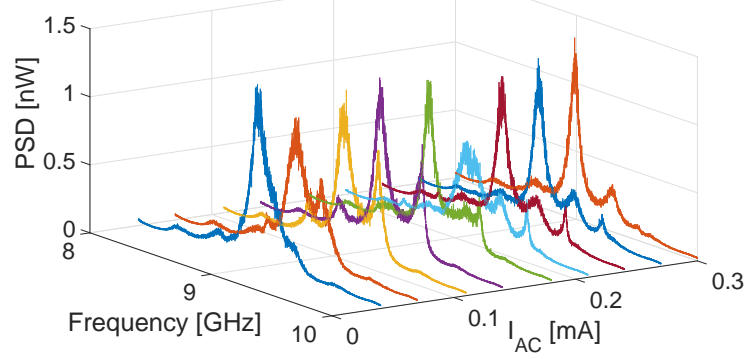

Fig. 6: Output frequency spectra in dependence of $I_{A C}$ for $H$ $=780 \mathrm{Oe}, \mathrm{I}_{\mathrm{DC}}=1.2 \mathrm{~mA}$ and data rate $=20 \mathrm{Mbit} / \mathrm{s}$

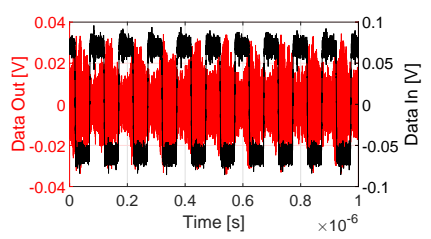

(a)

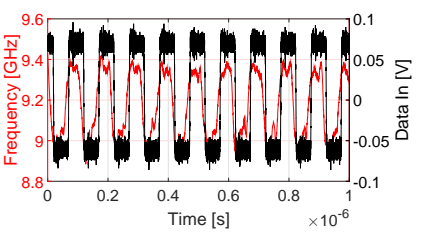

(b)
Fig. 7: (a) STNO output with 8 to $10 \mathrm{GHz}$ numerical filtering (b) STNO frequency time trace after 1000 points averaging

that the frequency is modulated between $9 \mathrm{GHz}\left(\mathrm{I}_{\mathrm{STNO}}=\mathrm{I}_{\mathrm{DC}}\right.$ $=1.2 \mathrm{~mA})$ and $9.38 \mathrm{GHz}\left(\mathrm{I}_{\mathrm{STNO}}=\mathrm{I}_{\mathrm{DC}}-\mathrm{I}_{\mathrm{AC}}=1.06 \mathrm{~mA}\right)$ in agreement with the $f$ versus $\mathrm{I}_{\mathrm{AC}}$ curve of figure 6 . This clearly demonstrates the BFSK modulation for the data rate of $20 \mathrm{Mbit} / \mathrm{s}$.

\section{CONCLUSION}

We have demonstrated the first BFSK modulation card using STNOs via current modulation. Frequency modulation with a frequency shift around $380 \mathrm{MHz}$ was observed when the current flowing through the STNO was shifted between 1.2 $\mathrm{mA}$ and $1.06 \mathrm{~mA}$ with a maximal data rate of $20 \mathrm{Mbit} / \mathrm{s}$. Thus, the feasibility of the BFSK modulation using STNOs was proved. However, some challenges must be overcome in order to lift this concept to the application level. The STNOs have to be designed to enable much more output power and smaller linewidth, in order that the design of its receiver can be simple and applicable for modern wireless systems.

\section{ACKNOWLEDGMENT}

The research leading to these results has received funding from (FP7-ICT-2011-8) under grant agreement N.317950 (MOSAIC) and (H2020-ICT-2015) under grant agreement N.687973 (GREAT). The authors gratefully thank HGST Inc. for providing the MTJ STNO devices.

\section{REFERENCES}

[1] M. R. Stan, M. Kabir, S. Wolf, and J. Lu, "Spin torque nano oscillators as key building blocks for the systems-on-chip of the future," in 2014 IEEE/ACM International Symposium on Nanoscale Architectures (NANOARCH), July 2014, pp. 37-38.

[2] M. Quinsat, F. Garcia-Sanchez, A. S. Jenkins, V. S. Tiberkevich, A. N. Slavin, L. D. Buda-Prejbeanu, A. Zeltser, J. A. Katine, B. Dieny, M.-C. Cyrille, and U. Ebels, "Modulation bandwidth of spin torque oscillators under current modulation," Applied Physics Letters, vol. 105, no. 15, p. 152401, 2014. [Online]. Available: http://dx.doi.org/10.1063/1.4898093

[3] M. Manfrini, T. Devolder, J.-V. Kim, P. Crozat, C. Chappert, W. V. Roy, and L. Lagae1, "Frequency shift keying in vortex-based spin torque oscillators," Materials Science; Mesoscale and Nanoscale Physics, March 2011.

[4] H. S. Choi, S. Y. Kang, S. J. Cho, I. Y. Oh, M. Shin, H. Park, C. Jang, B. C. Min, S. I. Kim, S. Y. Park, and C. S. Park, "Spin nano oscillator based wireless communication," Scientific Reports, vol. 4, no. 1, pp. 214 223, June 2014.

[5] R. Sharma, P. Duerrenfeld, M. Ranjbar, R. K. Dumas, J. Akerman, and P. K. Muduli, "Modulation rate study in a spin-torque oscillatorbased wireless communication system," IEEE Transactions on Magnetics, vol. 51, no. 11, pp. 1-4, Nov 2015.

[6] I. Y. Oh, S. Y. Park, D. H. Kang, and C. S. Park, "Wireless spintronics modulation with a spin torque nano-oscillator (stno) array," IEEE Microwave and Wireless Components Letters, vol. 24, no. 7, pp. 502-504, July 2014. 